\section{Glucanase Inhibitor Protein (GIP)}

\author{
Altino Choupina $^{1}$ and Ivone M. Martins ${ }^{2}$ \\ ${ }^{1}$ CIMO-Mountain Research Center, Agricultural \\ College of Bragança, Polytechnic Institute of \\ Bragança, Bragança, Portugal \\ ${ }^{2} \mathrm{CEB}$ - Centre of Biological Engineering, \\ University of Minho, Braga, Portugal
}

\section{Synonyms}

Chymotrypsin (S1); Serine protease homologs (SPH)

\section{Historical Background}

Several key cellular events, such as adhesion to the host surface, penetration, and colonization of host tissue, take place during plant infection by oomycetes that can also manipulate biochemical and physiological processes in their host plants through a diverse array of virulence or avirulence molecules, known as effectors (Birch et al. 2006; Ellis et al. 2006; Kamoun 2007; Schornack et al. 2009). In susceptible plants, these effectors promote infection by suppressing defense responses, enhancing susceptibility, or inducing disease symptoms. In resistant plants, the products of the resistance genes are able to recognize the effectors, promoting an efective defense response known as hypersensitive response (HR) which restricts the pathogen to an area of scorched earth besides host cell death (Kamoun 2003; Kamoun 2007; Schornack et al. 2009). Phytophthora effectors that suppress host defense responses have been described in several pathosystems (Kamoun et al. 1999; Day and Graham 2007; Attard et al. 2008). Suppression of host defenses can occur through the production of inhibitor proteins that target host enzymes (Valueva and Mosolov 2004). Early observations that plants secrete inhibitor proteins that bind and inactive microbial hydrolases, specifically the binding of plant polygalacturonase inhibitor proteins (PGIPs) to fungal polygalacturonases, spurred the search for analogous inhibitor proteins from microbes that might inactivate the $\beta(1,3)$ endoglucanases or chitinases of the plant host (Rose et al. 2002). Glucanase inhibitor protein (GIP) is thought to function as counter defensive molecule that inhibits the degradation of $\beta(1,3)$ and $\beta(1,6)$ glucans in the pathogen cell wall and/or the release of defense-eliciting oligosaccharides by host $\beta(1,3)$ endoglucanases (Kamoun 2003). Pathogens secrete $\beta(1,4)$ endoglucanases, which hydrolyze a range of plant cell wall glucans, while the host counters by synthesizing $\beta(1,3)$ endoglucanase that specifically degrade the hyphal wall of $\beta(1,3)$ and $\beta(1,6)$ glucans, rendering the pathogen susceptible to cell lysis. The $\beta(1,3)$ and $\beta(1,6)$ glucan elicitors that are released during this process are perceived by the plant, by a presumed plasma membrane-bound receptor, and a signaling cascade is initiated, resulting in the up-regulation of a number of plant defenses. Since the recent identification of GIPs, studies have focused on their biochemical characterization, addressing features such as binding properties and ligand specificity. The observation that these proteins bind strongly to enzymes that degrade cell wall polysaccharides, together with various sets of expression data, suggests that they are likely to influence plant-pathogen interactions. However, their biological significance has not yet been established. Many questions also remain at the molecular level, such as the identity of the domains 


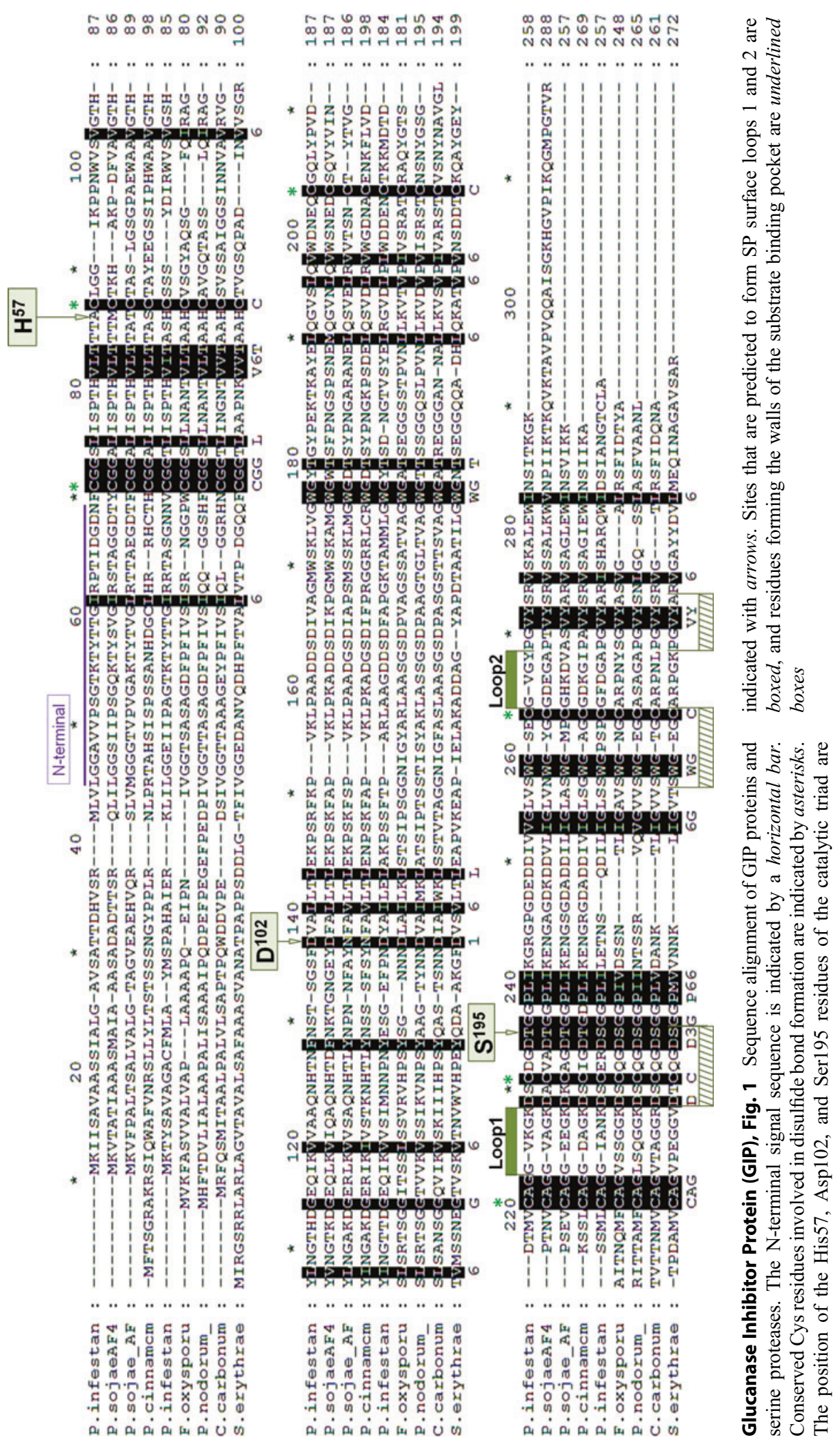


and key residues of the inhibitor proteins that contribute to the recognition specificity and high avidity binding.

\section{GIP's Homology}

A BLAST search of the databases revealed that the GIPs protein sequences are homologous with the chymotrypsin subclass of serine proteases (SP). The overall amino acid sequence homology of the GIPs with a number of chymotrypsins was similar to that typically observed among SP (20-40\%), and a number of characteristic features of SP were also present, including many sequence motifs and six cysteine residues (Fig. 1). A characteristic feature of SP is the catalytic triad charge relay system, referred to as His-57, Asp-102, and Ser-195 that are essential for the proteolytic function. However, this intact "catalytic triad" is not present in none of the predicted GIPs (Rose et al. 2002). Therefore, while GIPs strongly resemble SP, they are not expected to function as proteases. The triad of Phytophtora cinnamomi GIP is Ser-57, Asn-102, and Thr-195. Although GIPs are proteolytically inactive, several stretches of amino acids and motifs that are highly conserved among SP are present in the GIPs. Firstly, an N-terminal signal sequence is present that targets the enzyme for secretion. Secondly, GIPs possess three characteristically spaced pairs of Cys residues that are involved in the formation of disulfide bonds. Thirdly, key functional residues are present in the S1A specific substrate binding pocket and the two regions that flank the catalytic residues in the binding pocket form so-called variable surface loops 1 and 2. These surface loops contribute to the geometry of the SP specificity pocket and act synergistically to influence selective substrate binding and catalysis. A phylogenetic analysis of the GIP sequences aligned with other S1A clan SP from a number of evolutionarily diverse organisms revealed that the GIPs form a distinct group (Fig. 1).

\section{GIP's Structure}

The substantial amount of literature related to SP structure and function provides a valuable opportunity to make structural predictions about GIPs and to identify regions of the proteins that are involved in binding to the endoglucanases ligands or that may dictate ligand specificity (Fig. 2).

The overall geometry of the two classes of proteins is clearly predicted to be very similar and the software analysis designated the structure as a high confidence prediction. The model in Fig. 2c assumes that the inhibitor uses a trypsinlike mechanism of recognition (York et al. 2004) whereby GIP identifies a Lys residue on the surface of the glucanase molecule.

GIPs represent the first example of proteolytically inactive SP homologs that function as enzyme inhibitors. It can be hypothesized that a major role for GIP is to suppress the release of glucan elicitors during P. cinnamomi infection,

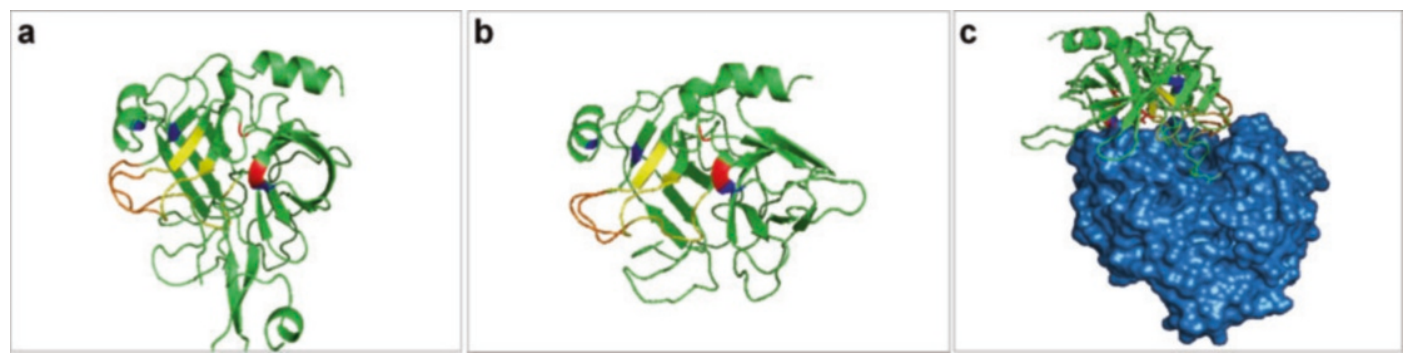

Glucanase Inhibitor Protein (GIP), Fig. 2 (a) The crystal structure of $\mathrm{R} 117 \mathrm{H}$ mutant rat anionic trypsin complexed with bovine pancreatic trypsin inhibitor BPTI (PDB 1C07) was used as a template to predict the corresponding structure of $P$. cinnamomi GIP; (b) using a computational approach (ESyPred3D), the catalytic triad of trypsin and the equivalent residues of GIP are colored: red catalytic triad, blue conserved Cys residues, yellow residues forming the walls of the substrate binding pocket, orange amino acids predicted to form surface loops1 and 2; (c) a model of an $\beta$-endoglucanase (PDB 1AQ0) docking with P. cinnamomi GIP 
thereby reducing the effectiveness of the plant host's surveillance system (York et al. 2004; Damasceno et al. 2008; Liu et al. 2010).

\section{Summary}

In order to inhibit the activity of plant extracellular enzymes, $\quad \beta(1,3)$ and $\beta(1,6)$ endoglucanases (EGases), involved in plant defense responses, including during the infection process, glucanase inhibitor proteins (GIPs) are secreted by species of the genus Phytophthora. Besides the structural homology to the chymotrypsin class of serine proteases (SP), GIPs, due to the absence of an intact catalytic triad, lack proteolytic activity. For that reason, they belong to a broader class of nonfunctional proteins caled serine protease homologs (SPH). Despite the high homology of GIPs to the SP subfamily S1A, some questions remain regarding the expression patterns and potential roles of different GIPs during pathogenesis and also their possible interaction with host EGases in the plant apoplast.

\section{References}

Attard A, Gourgues M, Galiana E, Panabieres F, Ponchet M, Keller H. Strategies of attack and defense in plant-oomycete interactions, accentuated for Phytophthora parasitica Dastur (syn. P. Nicotianae Breda de Haan). J Plant Physiol. 2008;165(1):83-94.

Birch PR, Rehmany AP, Pritchard L, Kamoun S, Beynon JL. Trafficking arms: oomycete effectors enter host plant cells. Trends Microbiol. 2006;14(1): $8-11$.

Damasceno CM, Bishop JG, Ripoll DR, Win J, Kamoun S, Rose JK. Structure of the glucanase inhibitor protein (GIP) family from phytophthora species suggests coevolution with plant endo-beta-1,3-glucanases. Mol Plant-Microbe Interact. 2008;21(6):820-30.

Day B, Graham T. The plant host pathogen interface: cell wall and membrane dynamics of pathogen-induced responses. Ann NY Acad Sci. 2007;1113:123-34.

Ellis J, Catanzariti AM, Dodds P. The problem of how fungal and oomycete avirulence proteins enter plant cells. Trends Plant Sci. 2006;11(2):61-3.

Kamoun S. Molecular genetics of pathogenic oomycetes. Eukaryot Cell. 2003;2(2):191-9.

Kamoun S. Groovy times: filamentous pathogen effectors revealed. Curr Opin Plant Biol. 2007;10(4):358-65.
Kamoun S, Huitema E, Vleeshouwers VG. Resistance to oomycetes: a general role for the hypersensitive response? Trends Plant Sci. 1999;4(5):196-200.

Liu B, Xue X, Cui S, Zhang X, Han Q, Zhu L, Liang X, Wang X, Huang L, Chen X, Kang Z. Cloning and characterization of a wheat beta-1,3-glucanase gene induced by the stripe rust pathogen Puccinia striiformis f. sp. tritici. Mol Biol Rep. 2010;37(2):1045-52.

Rose JK, Ham KS, Darvill AG, Albersheim P. Molecular cloning and characterization of glucanase inhibitor proteins: coevolution of a counterdefense mechanism by plant pathogens. Plant Cell. 2002;14:1329-45.

Schornack S, Huitema E, Cano LM, Bozkurt TO, Oliva R, Van Damme M, Schwizer S, Raffaele S, ChaparroGarcia A, Farrer R, Segretin ME, Bos J, Haas BJ, Zody MC, Nusbaum C, Win J, Thines M, Kamoun $\mathrm{S}$. Ten things to know about oomycete effectors. Mol Plant Pathol. 2009;10(6):795-803.

Valueva TA, Mosolov VV. Role of inhibitors of proteolytic enzymes in plant defense against phytopathogenic microorganisms. Biochemistry (Mosc). 2004;69(11):1305-9.

York WS, Qin Q, Rose JK. Proteinaceous inhibitors of endo-beta-glucanases. Biochim Biophys Acta. 2004;1696(2):223-33. 